\title{
Imaging Single Tumor Cells in Mice Using Multimodal Correlative Microscopy
}

M.A. Karreman $^{1}$, L. Mercier ${ }^{2-5}$, N.L. Schieber ${ }^{1}$, G. Solecki ${ }^{6}$, G. Allio ${ }^{2-5}$, F. Winkler ${ }^{6}$, B. Ruthensteiner ${ }^{7}$, J.G. Goetz ${ }^{2-5}$ and Y. Schwab ${ }^{1}$

1. Cell Biology and Biophysics, EMBL Heidelberg, Heidelberg, Germany.

${ }^{2 .}$ MN3T, Inserm U1109, Strasbourg, 67200, France.

${ }^{3 .}$ Université de Strasbourg, Strasbourg, 67000, France.

${ }^{4 .}$ LabEx Medalis, Université de Strasbourg, Strasbourg, 67000, France.

5. Fédération de Médecine Translationnelle de Strasbourg (FMTS), Université de Strasbourg, Strasbourg, 67000, France.

6. Clinical Cooperation Unit Neurooncology, German Cancer Research Center (DKFZ), Heidelberg, Germany.

${ }^{7}$ Evertebrata Varia, Zoologische Staatssammlung München, Munich 81247, Germany

Metastasis is the main cause of cancer-related mortality, but how tumor cells spread through the tissue in vivo is still largely unknown. Intravital microscopy (IVM) enables studying crucial steps of the metastatic process [1], but it is limited in resolution and it fails to reveal the structural context. Combining IVM to 3D Electron Microscopy (3DEM) enables to correlate functional and dynamic in vivo imaging to high-resolution of the tumor cells and their microenvironment. However, keeping track of single tumor cells when moving from IVM to EM imaging is highly challenging in complex tissue samples [2].

We have developed a method that exploits x-ray microscopic computer tomography (microCT) [3] to correlate IVM to EM [4]. First, fluorescent tumor cells are xenotransplanted to living mice, and monitored by IVM. 3D datasets of the tumor cells and the surrounding fluorescently stained vasculature are obtained. Next, the region of interest is processed for EM and embedded in resin. The sample is then scanned with microCT, revealing the outlines of the resin block, the biopsy and the vasculature within. Using 3D imaging software, the IVM and microCT volumes are registered, enabling to map the position of the tumor cell within the resin-embedded specimen. Finally, targeted trimming enables to quickly approach the tumor cell inside the resin block and expose it for 3D imaging with FIB-SEM or electron tomography.

The method will be demonstrated on capturing arrested tumor cells in the vasculature of mouse brain samples $[4,5]$, and on migrating invasive tumor cells in mouse skin $[2,4]$. Enabling to predict the position of the tumor cell within the resin block with an accuracy of $>5 \mu \mathrm{m}$ significantly speeds up the process of correlating IVM to EM; from several months to $\sim 2$ weeks. The correlative approach uniquely enables multiple high-resolution observations of rare metastatic events in tissue, allowing obtaining statistically relevant conclusions on the crucial steps in the dissemination of tumor cells [4].

References:

[1] CLGJ Scheele, C Maynard and J Van Rheenen, Trends in Cancer 2 (2016), p 205-216.

[2] MA Karreman et al. PLoS One 9 (2014), e114448.

[3] S Handschuh et al. Front Zoology 10 (2013), p 1-16.

[4] MA Karreman et al. Journal of Cell Science 129 (2016), p. 444-456

[5] Y Kienast, L von Baumgarten, M Fuhrmann, WE Klinkert, R Goldbrunner, J Herms, F Winkler. Nature Medicine 16 (2010), p 116-122. 


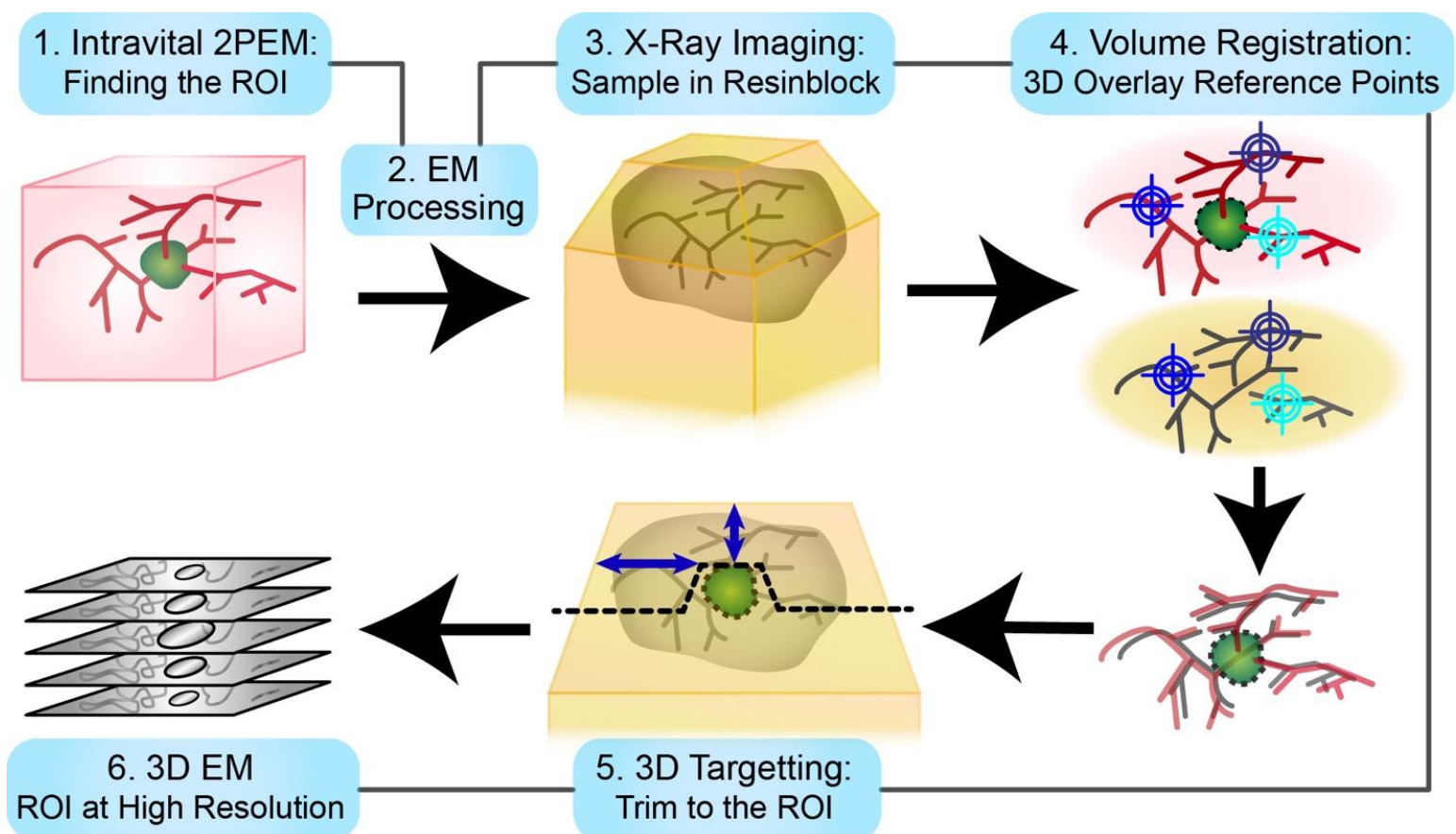

Figure 1. Multimodal Correlative Microscopy combines intravital imaging, $x$-ray microCT and electron microscopy in an efficient workflow to image single tumor cells in vivo and, next, at high resolution in $3 \mathrm{D}[4]$.

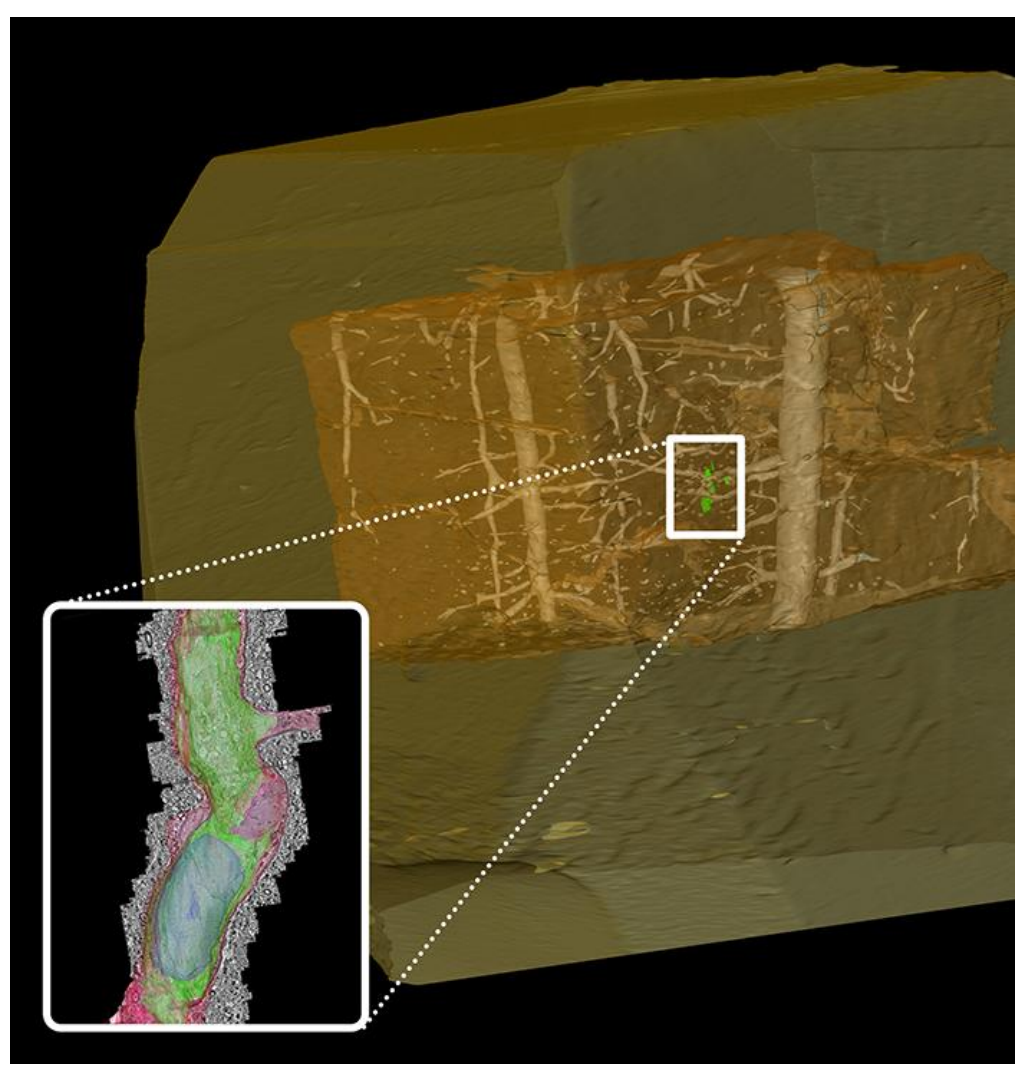

Figure 2. Multimodal Correlative Microscopy enables to predict the position of single tumor cells inside brain biopsies that processed for EM and embedded in resin. In this way, it is possible to quickly retrieve the tumor cell inside the sample and image it with Focussed Ion Beam Scanning Electron Microscopy [4]. 